\title{
EVERY ZERO-DIMENSIONAL SPACE IS CELL SOLUBLE
}

\author{
TOSHIJI TERADA
}

(Communicated by Dennis Burke)

\begin{abstract}
In his study of the question of representing a space as a retract of a homogeneous space, Arhangel'skii introduced an interesting topological property called cell solubility. He raised the following problem: Is every zerodimensional compact space cell soluble? We will give an affirmative answer to this problem.
\end{abstract}

\section{INTRODUCTION}

A topological space $X$ is called homogeneous if for arbitrary points $x, y \in X$ there exists a homeomorphism $f$ from $X$ onto itself such that $f(x)=y$. Since topological groups are homogeneous and any power $S^{\kappa}$ of the circle $S$ is a compact topological group, every Tychonoff space is embedded in some homogeneous compact $T_{2}$-space. On the other hand, Motorov (see [1] or [2]) showed that there exists a metrizable compact space that is not a retract of any homogeneous compact $T_{2}$-space. When is a compact $T_{2}$-space $X$ a retract of some homogeneous compact $T_{2}$-space? Concerning this problem, Arhangel'skii found an interesting topological property that every retract of an arbitrary homogeneous compact $T_{2}$-space possesses.

Let $q=(Y, Z, \mathscr{E})$ be a triple, where $Y$ is a topological space, $Z$ a subspace of $Y$, and $\mathscr{E}$ a nonempty family of subsets of $Y$. Let $X$ be an arbitrary topological space. A closed subset $P$ of $X$ is said to be $q$-saturated if for any continuous map $f: Y \rightarrow X$ such that $f(L) \cap P \neq \varnothing$ for all $L \in \mathscr{E}$ we have $f(Z) \subset P$. For an arbitrary point $x \in X$ we denote by $F_{q}(X)$ the intersection of all $q$-saturated subsets containing $x$. The family $\left\{F_{q}(x): x \in X\right\}$ is called the cellularity induced by the triple $q$. The sets $F_{q}(x)$ are called the terms of the cellularity. The cellularity $\left\{F_{q}(x): x \in X\right\}$ is called disjoint if for all $x, y \in X$ either $F_{q}(x)=F_{q}(y)$ or $F_{q}(x) \cap F_{q}(y)=\varnothing$. A topological space $X$ is called cell soluble if for any triple $q$ (as above), its induced cellularity is disjoint, provided that at least one of its terms is compact (see [1] or [2]).

Arhangel'skii proved that every retract of an arbitrary homogeneous compact $T_{2}$-space is cell soluble. Moreover, he raised the following problem.

Received by the editors May 31, 1989 and, in revised form, October 30, 1989.

1980 Mathematics Subject Classification (1985 Revision). Primary 54C99; Secondary 54F45.

Key words and phrases. Homogeneous, zero-dimensional, retract, cell soluble. 
Problem. Is every zero-dimensional compact $T_{2}$-space cell soluble?

In this note we will give an affirmative answer to this problem.

\section{Conclusion}

We recall that a $T_{1}$-space $X$ is called zero-dimensional if $X$ has an open base consisting of clopen subsets.

Theorem. Every zero-dimensional topological space is cell soluble.

Proof. Let $X$ be a zero-dimensional topological space. Let $q=(Y, Z, \mathscr{E})$ be an arbitrary triple, where $Y$ is a topological space, $Z$ a subspace of $Y$, and $\mathscr{E}$ a nonempty family of subsets of $Y$. If $Z=\varnothing$ and $f: Y \rightarrow X$ is continuous, then $F_{q}(x)=\{x\}$ for any $x \in X$ since $f(\varnothing)=\varnothing \subset\{x\}$. Hence we can assume that $Z \neq \varnothing$.

Suppose that there exists a clopen subset $G$ of $Y$ such that $G \cap Z \neq \varnothing$ and $L \backslash G \neq \varnothing$ for every $L \in \mathscr{E}$. Then we can show that $F_{q}(x)=X$ for any $x \in X$. In fact, if a subset $P$ of $X$ satisfies $P \neq X$, then there exists a continuous map $f: Y \rightarrow X$ such that $f(G) \subset X \backslash P$ and $f(Y \backslash G) \subset P$. This shows that $P$ is not $q$-saturated.

The case remaining is the following: For any clopen subset $G$ of $Y$, if $G \cap Z \neq \varnothing$, then there exists some member $L$ of $\mathscr{E}$ such that $L \subset G$. In this case, it will be proved that $F_{q}(x)=\{x\}$ for any $x \in X$. Let $f: Y \rightarrow X$ be a continuous map such that $f(L) \cap\{x\} \neq \varnothing$ for any $L \in \mathscr{E}$. Then it suffices to show that $f(Z)=\{x\}$. If $f(Z) \neq\{x\}$, then there exists a clopen set $U$ of $X$ such that $U \cap f(Z) \neq \varnothing$ and $x \notin U$ are satisfied. But this is a contradiction since the clopen subset $f^{-1}(U)$ satisfies $f^{-1}(U) \cap Z \neq \varnothing$ and $L \backslash f^{-1}(U) \neq \varnothing$ for any $L \in \mathscr{E}$. This completes the proof.

Remark 1. From our result it follows that another problem of Arhangel'skii [2] is solved simultaneously.

Problem. Is it true that the space $\beta N \backslash N$ (respectively, the growth of the space $\beta(\tau))$ is cell soluble?

Remark 2. Van Douwen [3] posed the following very interesting problem.

Problem. Is a compact $T_{2}$-space nonhomogeneous if it can be mapped continuously onto $\beta N$ ?

It is well known that this problem is equivalent to the following:

Problem. Is $\beta N$ the retract of some homogeneous compact $T_{2}$-space?

Our result shows that a technique like that of Motorov is not appropriate for solving the last problem. 


\section{REFERENCES}

1. A. V. Arhangel'skii, Cell structures and homogeneity, Mat. Zametki 37 (1985), 580-586; Math. Notes 37 (1985), 321-324.

2. __ Topological homogeneity. Topological groups and their continuous images, Uspekhi Mat. Nauk 2 (1987), 69-105; Russian Math. Surveys 2 (1987), 83-131.

3. E. K. van Douwen, Non-homogeneity of products of preimages and $\pi$-weight, Proc. Amer. Math. Soc. 69 (1978), 183-192.

Department of Mathematics, Faculty of Engineering, Yokohama National UniverSity, 156 TokiwadaI, Hodogaya, Yokohama, Japan 